\title{
Distribution of Static Normal Reactions to Wheels of Open-Pit Dump Trucks Depending on the Longitudinal and Cross Sections of the Open-Pit Road
}

\author{
Michail Dadonov ${ }^{1}$, Alexander Kulpin ${ }^{1,}$, Oleg Ostanin ${ }^{1}$ and Erkin Suleimenov ${ }^{2}$ \\ ${ }^{1}$ T.F. Gorbachev Kuzbass State Technical University, Kemerovo, Russian Federation \\ ${ }^{2}$ Kazakh Humanitarian-Law Innovative University, Semey, Kazakhstan
}

\begin{abstract}
Vehicle costs for rock mass transportation are more than $50 \%$ of total costs, among which $25-30 \%$ are tire costs. The average tire life in mining enterprises is lower than that the value recommended by production plants. The tire life of open-pit dump trucks is more dependent on the normal load on the wheel. The main factor in the change of the normal load is location of the wheel on the dump truck and the open-pit road's section. Determination of the maximum loaded tire in different operating conditions will allow increasing the tire life and preventing its premature failure. The TKPH index (tire performance) recommended by tire manufacturers does not take into account the diversity of mining conditions and variations of sections of open-pit roads on different routes. The average load per trip is defined as movement of a loaded open-pit truck and an empty open-pit truck on a straight reach of a road. The proposed method for recording of redistribution of the load on the wheel allows making adjustments to recording of TKPH indexes and thereby effectively managing the tire life, which in turn will make it possible to more fully use the tire life, increase its reliability and reduce the cost of rock mass transportation.
\end{abstract}

\section{Introduction}

Tires are one of the most important parts of modern open-pit dump trucks, because they have a significant impact on traffic safety, performance and workings, and they also form one of the largest cost items associated with operation $[1,2]$.

One of the most significant operational factors affecting the tire life of dump trucks is the normal tire load and its distribution depending on location of wheels. The ability to determine the most loaded tire in various road conditions will help to develop and implement measures to prevent the reduction of its life and premature failure $[3,4]$.

\footnotetext{
${ }^{*}$ Corresponding author: kag.ea@mail.ru
} 


\section{Results and discussion}

The value of the normal tire load is estimated by the value of the normal reaction that occurs in the contact patch of the wheel with the support surface [5, 6]. Figures 1 and 2 show forces and reactions operating on a dump truck.

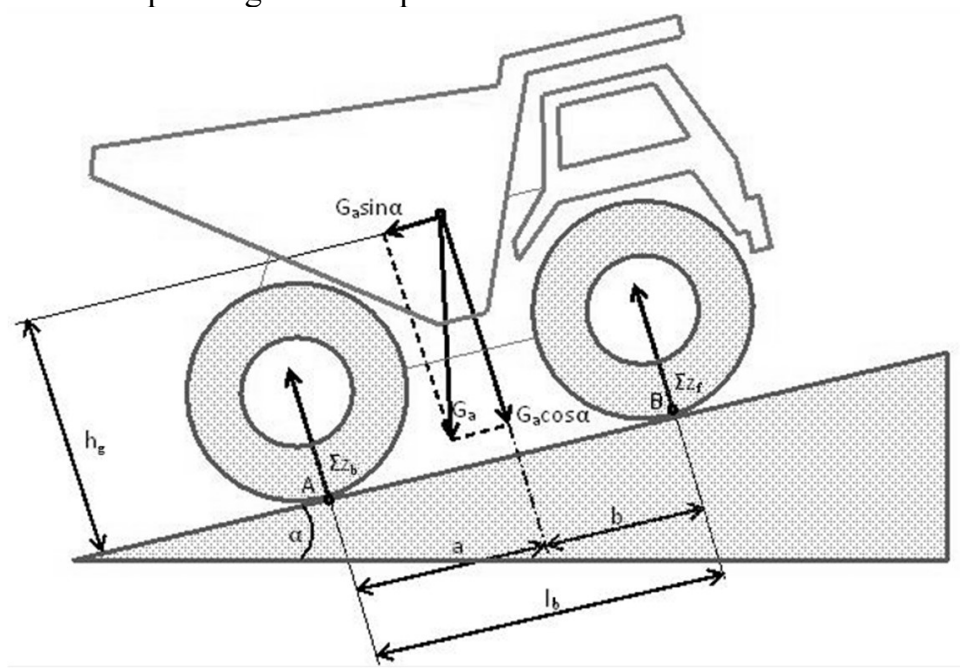

Fig. 1. Forces and reactions operating on a dump truck in the longitudinal plane.

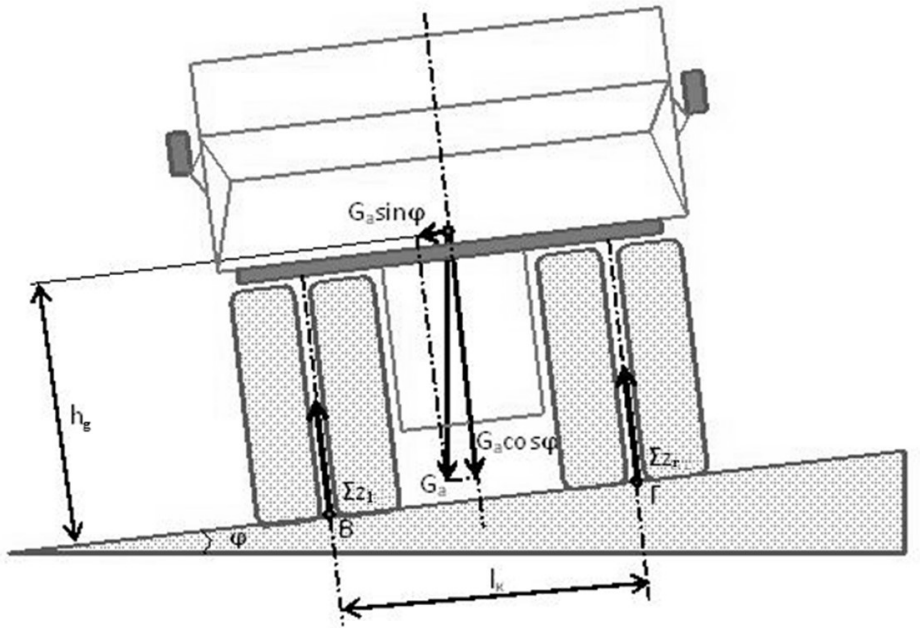

Fig. 2. Forces and reactions operating on a dump truck in the transverse plane.

Where $G_{a}-$ Weight dump truck, $H$, equal to the sum of the dumper's own weight and the actual weight of the load;

$\Sigma z_{b}, \Sigma z_{f}-$ the sum of the normal reactions of the rear and front wheels respectively

$\Sigma z_{l}, \Sigma z_{r}$ - the sum of the normal reactions of the left and right wheels respectively

$h_{g}$ - height of centre of gravity dump truck, m;

$l_{b}$ - distance apart front and back-axle

$l_{k}-$ wheel track

$a$ - centre-to-centre distance heavy and back axle dump truck

$b$-centre-to-centre distance heavy and forward axle dump truck

$\alpha$ - longitudinal profile angle of a career road 
$+\alpha$ - upgrading

$-\alpha-$ downhill operation

$\varphi$ - career road cross-section angle

To determine normal reactions, we compose the equation of force moments relative to points A, B, C, D.

$$
\begin{gathered}
\Sigma M_{A}=a G_{a} \cos \alpha-h_{g} G_{a} \sin \alpha-(a+b) \Sigma z_{f} ; \\
\Sigma M_{B}=(a+b) \Sigma z_{b}-b G_{a} \cos \alpha-h_{g} G_{a} \sin \alpha ; \\
\Sigma M_{C}=\frac{l_{r}}{2} G_{a} \cos \varphi-h_{g} G_{a} \sin \varphi-l_{r} \Sigma z_{r} ; \\
\Sigma M_{D}=l_{r} \Sigma z_{l}-\frac{l_{r}}{2} G_{a} \cos \varphi-h_{g} G_{a} \sin \varphi .
\end{gathered}
$$

Based on the equations of moments, we express the sum of normal reactions of the front and back axles, the right and left sides.

$$
\begin{gathered}
\Sigma Z_{f}=\frac{a G_{\mathrm{a}} \cos \alpha-h_{g} G_{\mathrm{a}} \sin \alpha}{(a+b)} ; \\
\Sigma Z_{b}=\frac{b G_{a} \cos \alpha+h_{g} G_{a} \sin \alpha}{(a+b)} ; \\
\Sigma Z_{r}=\frac{\frac{l_{r}}{2} G_{a} \cos \varphi-h_{g} G_{a} \sin \varphi}{l_{r}} ; \\
\Sigma Z_{l}=\frac{\frac{l_{r}}{2} G_{a} \cos \varphi+h_{g} G_{a} \sin \varphi}{l_{r}} .
\end{gathered}
$$

When a dump truck is on a flat area of the road, i.e. when $\alpha=0, \varphi=0$

$$
\begin{gathered}
\Sigma z_{b}=G_{a} \frac{b}{a+b} \\
\Sigma z_{f}=G_{a} \frac{a}{a+b} \\
\Sigma z_{l}=\Sigma z_{r}=\frac{G_{a}}{2} .
\end{gathered}
$$

$\frac{a}{a+b}=\lambda_{f}$ is the weight percentage of the dump truck to the front axle on a flat surface.

$\frac{b}{a+b}=\lambda_{b}$ is the weight percentage of the dump truck to the back axle on a flat surface.

Given that the value of the longitudinal slope angle of the open-pit road does not exceed $\alpha \leq 140 \%\left(7.97^{\circ}\right)$, and the value of the transverse slope angle of the open-pit road does not exceed $\phi \leq 30 \%\left(1.72^{0}\right)$, it is conceivable that: $\cos \alpha \approx 1, \sin \alpha \approx \operatorname{tg} \alpha \approx \alpha(\mathrm{rad}), \cos \phi \approx 1, \sin \phi$ $\approx \operatorname{tg} \phi \approx \phi(\mathrm{rad})$.

Then, provided that $\alpha \neq 0$ and $\varphi \neq 0$, total normal reactions of the back and front axles, the left and right sides are determined as follows: 


$$
\begin{gathered}
\Sigma z_{b}=\frac{b G_{a}+h_{g} G_{a} \alpha}{a+b}=G_{a}\left(\frac{b}{a+b}+\frac{h_{g} \alpha}{a+b}\right)=G_{a} \lambda_{b}\left(1+\frac{h_{g} \alpha}{b}\right) ; \\
\Sigma z_{f}=\frac{a G_{a}-h_{g} G_{a} \alpha}{a+b}=G_{a}\left(\frac{a}{a+b}-\frac{h_{g} \alpha}{a+b}\right)=G_{a} \lambda_{f}\left(1-\frac{h_{g} \alpha}{a}\right) ; \\
\Sigma z_{l}=\frac{\frac{l_{r}}{2} G_{a}+h_{g} G_{a} \varphi}{l_{r}}=G_{a}\left(\frac{\frac{l_{r}}{2}+h_{g} \varphi}{l_{r}}\right)=\frac{G_{a}}{2}\left(1+\frac{2 h_{g} \varphi}{l_{r}}\right) ; \\
\Sigma Z_{r}=\frac{\frac{l_{r}}{2} G_{a}-h_{g} G_{a} \varphi}{l_{r}}=G_{a}\left(\frac{\frac{l_{r}}{2}-h_{g} \varphi}{l_{r}}\right)=\frac{G_{a}}{2}\left(1-\frac{2 h_{g} \varphi}{l_{r}}\right) .
\end{gathered}
$$

Analysis of all obtained equations shows that the multiplier in front of brackets represents total normal reactions of the back and front axles, the left and right sides, occurring on a flat platform with $\alpha=0$ and $\phi=0$. In this case, multipliers in brackets are load change coefficients depending on the longitudinal and cross sections of the road [7].

Load change factors for positive values of angles $\alpha>0$ and $\varphi>0$ (see Figures 1,2) are defined as follows:

$$
\begin{aligned}
& k_{b}=\lambda_{b}\left(1+\frac{h_{g} \alpha}{b}\right) ; \\
& k_{f}=\lambda_{f}\left(1-\frac{h_{g} \alpha}{a}\right) ; \\
& k_{l}=\frac{1}{2}\left(1+\frac{2 h_{g} \varphi}{l_{r}}\right) ; \\
& k_{r}=\frac{1}{2}\left(1-\frac{2 h_{g} \varphi}{l_{r}}\right) .
\end{aligned}
$$

For negative values of angles $\alpha<0$ and $\varphi<0$, the signs in formulas for calculation of load change factors should be changed to the opposite ones [8].

Using these factors, you can determine the value of the normal reaction to each wheel.

$$
\begin{aligned}
& z_{b-l}=G_{a} k_{b} k_{l} ; \\
& z_{b-r}=G_{a} k_{b} k_{r} ; \\
& z_{f-l}=G_{a} k_{f} k_{l} ; \\
& z_{f-r}=G_{a} k_{f} k_{r} .
\end{aligned}
$$

\section{Conclusion}

Below is the weight distribution diagram given as an example of calculation for each wheel of the dump truck BelAZ-75131, which is in a state of full load, depending on angles of the longitudinal and cross sections of the open-pit road (Figure 3 ).

Diagram analysis shows the significant influence of angles of the open-pit road section on the load of dump truck wheels [9].

In the considered case: $\mathrm{W}_{\mathrm{bmax}}=0.446, \mathrm{~W}_{\mathrm{bmin}}=0.237, \mathrm{~W}_{\mathrm{fmax}}=0.262, \mathrm{~W}_{\mathrm{fmin}}=0.081$.

In other words, almost half of the entire mass of the dump truck can put load on the back wheel, while the opposite front wheel will be almost without load in boundary road conditions $[10]$. 


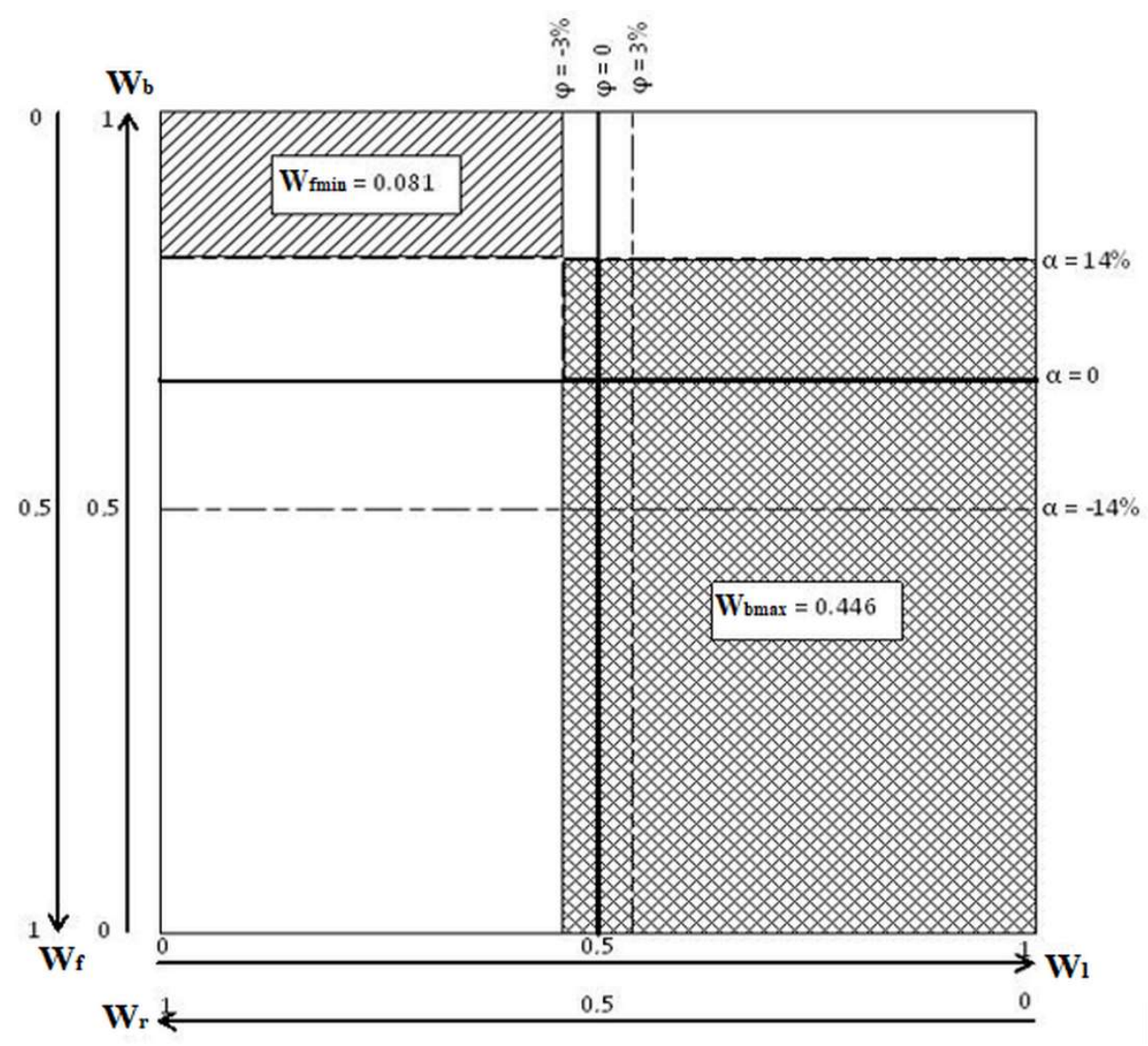

Fig. 3. Weight distribution diagram for each wheel of a dump truck BelAZ - 75131 with a total weight of 247 tons depending on angles of the longitudinal and cross sections of the open-pit road.

Taking into account a variety of mining operation conditions and section variants of openpit roads on different routes, it can be concluded that values of normal loads on the dump truck wheel that are average per route are not the same depending on the place of its installation [11]. Hence, it is necessary to make adjustments when determining the operational performance of tires, the TKPH index, depending on the longitudinal and cross sections of open-pit roads for more efficient management of the tire life of open-pit dump trucks [12]. At the same time, this index should be determined and adjusted for each wheel of the open-pit dump truck [13].

In addition, there is a need to determine the degree of influence of normal loads of the sum of instantaneous mass redistributions on average values, arising over time during movement of a dump truck along a given route [14,15].

\section{References}

1. A.G. Kulpin, D.V. Stenin, E.E. Kultayev, E.E. Kulpina, V.A. Borovtsov, Coal in the 21 st Century: Mining, Processing and Safety, 1, 68 (2016) 
2. D.V. Stenin, N.A Stenina, A.A Bakanov, Coal in the 21 st Century: Mining, Processing and Safety, 1, 54 (2016)

3. X Yang, O Olatunbosun, D Garcia-Pozuelo, E Bolarinwa, SAE Technical Papers, 7, 33 (2015)

4. Q. Sunqing, D. Junxiu, Ch. Guoxu, Lubricating Science, 11:3, 165 (1999)

5. A. A Samarskii, V. A Galaktionov, S. P Kurdiumov, A.P. Mikhailow, Blow-up in quasilinear parabolic equations (Walter de Gruytel, Berlin, 1995)

6. A. Abramovich, E Pudov, E Kuzin, E3S Web Conf., 21, 01011 (2017)

7. A Kvasova, B Gerike, E Murko, D Skudarnov, E3S Web Conf., 21, 03016 (2017)

8. F. Zhang, Z., Gu, S. Zhang, X. Ma, Zhu, Y. Zhendong yu Chongji, Journal of Vibration and Shock. 35:17, 71 (2016)

9. T.H. Langer, T.K. Iversen, O.O. Mouritsen, M.K. Bak, M.R. Hansen, International Journal of Vehicle Design, 65:2-3, 222 (2014)

10. J. Meech, J. Parreira, IFAC Proceedings Volumes, 15:1, 142 (2013)

11. Y. Li, W.Y. Liu, S. Frimpong, Engineering Failure Analysis, 23, 55 (2013)

12. R. Larson, J. Cuadrado, SAE International Journal of Commercial Vehicles, 5:1, 101 (2012)

13. K. Pal, T. Das, Polymer Engineering and Science, 48:12, 2410 (2008)

14. T. Eguchi, T. Muro, 15th International Conference of the International Society for Terrain Vehicle Systems, 89, 126 (2005)

15. P.F. Knights, A.L. Boerner, Mining Engineering, 53:8, 51 (2001) 